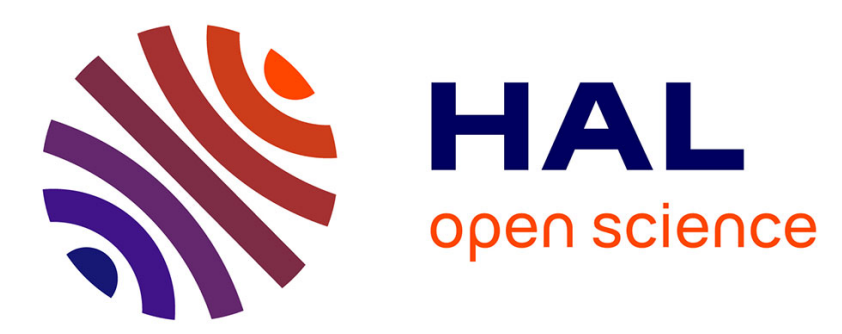

\title{
A discrete element model to investigate sub-surface damage due to surface polishing
}

\author{
Ivan Iordanoff, A Battentier, Jérôme Neauport, J.L. Charles
}

\section{To cite this version:}

Ivan Iordanoff, A Battentier, Jérôme Neauport, J.L. Charles. A discrete element model to investigate sub-surface damage due to surface polishing. Tribology International, 2008, 10.1016/j.triboint.2008.02.018 . cea-01217061

\section{HAL Id: cea-01217061 https://hal-cea.archives-ouvertes.fr/cea-01217061}

Submitted on 18 Oct 2015

HAL is a multi-disciplinary open access archive for the deposit and dissemination of scientific research documents, whether they are published or not. The documents may come from teaching and research institutions in France or abroad, or from public or private research centers.
L'archive ouverte pluridisciplinaire HAL, est destinée au dépôt et à la diffusion de documents scientifiques de niveau recherche, publiés ou non, émanant des établissements d'enseignement et de recherche français ou étrangers, des laboratoires publics ou privés. 


\title{
A discrete element model to investigate sub-surface damage due to surface polishing
}

\author{
Ivan Iordanoff $^{\mathrm{a}, *}$, A. Battentier ${ }^{\mathrm{a}}$, J. Néauport $^{\mathrm{b}}$, J.L. Charles ${ }^{\mathrm{a}}$ \\ ${ }^{a}$ ENSAM, LAMEFIP, Esplanade des Arts et Métiers, 33405 Talence Cedex, France \\ b Commissariat à l'Énergie Atomique, Centre d'Études Scientifiques et Techniques d'Aquitaine, BP 2, 33114 Le Barp, France
}

\section{A R T I C L E I N F O}

\section{Article history:}

Received 11 September 2007

Received in revised form

15 February 2008

Accepted 28 February 2008

\section{Keywords:}

Abrasion process

Sub-surface damage

Wear

DEM (discrete element method)

Optics

Surface finishing

\begin{abstract}
A B S T R A C T
Large high-power laser facilities such as megajoule laser (LMJ) or National Ignition Facility (NIF) are designed to focus about $2 \mathrm{MJ}$ of energy at the wavelength of $351 \mathrm{~nm}$, in the center of an experiment chamber. The final optic assembly of these systems, operating at $351 \mathrm{~nm}$ is made of large fused silica optics working in transmission. When submitted to laser at the wavelength of $351 \mathrm{~nm}$, fused silica optics can exhibit damage, induced by the high amount of energy traversing the part. The created damage is a set of micro-chips that appear on the optic surface. Current researches have shown that this damage could be initiated on pre-existing sub-surface damages created during the optics manufacturing process. It is then very important to understand, for various set of manufacturing parameters, what are the key parameters for sub-surface damage. The presented work details the development of a simplified model to investigate the polishing process. Both silica (the material to be polished) and the abrasive particles are modeled using a discrete element approach. This numerical tool allows following the evolution of micro-cracks inside the material during the abrasion process. It is shown how the mechanical properties (pressure), the abrasive properties (shape and quantity of abrasive particles) and the system properties (filtration) have an influence on the sub-surface properties at the end of the process.
\end{abstract}

(c) 2008 Elsevier Ltd. All rights reserved.

\section{Introduction}

When submitted to high fluences $\left(>5-10 \mathrm{~J} / \mathrm{cm}^{2}\right)$ at the wavelength of $351 \mathrm{~nm}$, transmission fused silica optics such as lenses, windows can exhibit damage similar to micro-chipping. Damage is induced by the high amount of energy traversing the part. An example of such laser damage is shown in Fig. 1.

Consequently, lifetime in the UV of fused silica optics used on large high-power laser facilities such as megajoule laser (LMJ) [1] or National Ignition Facility (NIF) [2] has been the subject of extensive studies. It has been shown that at the wavelength of $351 \mathrm{~nm}$, the lifetime of fused silica optics is determined by both surface damage initiation and damage growth [3]. Hence, strategies developed to improve lifetime rely on reducing initiator density and using mitigation techniques to inhibit growth of damage sites induced by residual initiators [4].

As for the nature of damage initiators, it has been shown that damage initiation can occur on local absorption of contaminants coming from polishing slurries buried into the fused silica optics interface [5]. Sub-surface cracks, such as the ones present in

\footnotetext{
* Corresponding author. Tel.: +33 556845391; fax: +33556845366.

E-mail address: ivan.iordanoff@lamef.bordeaux.ensam.fr (I. Iordanoff).
}

sub-surface damage (SSD) layer of conventionally polished optical parts, are also possible damage initiators [6,7]. SSD is created during the first steps of the optic manufacturing [8]. Sawing and grinding involve hard abrasive grains put in contact with the optical part to remove material in brittle mode. Generated cracks can then extend far below the surface. Loose abrasive grinding and polishing can also damage the optical surface as well. SSD depth is also greatly process dependent [9]. Creation of SSD during fused silica grinding and polishing can be seen as the action of multiple loaded hard particles on a brittle material. Therefore, most of the models developed to understand the creation of SSD is based on basic fracture relationship (single static indent, trailing indent, etc.) combined with experimental data. It leads to an estimation of a maximal depth of SSD for given manufacturing parameters (load, particle diameter, etc.) but does not give a complete understanding of the abrasion phenomenon. Our aim is to get a better physical understanding of the grinding process. Hence, the present work is based on the development of a three-dimensional discrete element simulation (DES) of the grinding step, an early step of the fused silica optic polishing process. This study focuses on SSD.

In 1995, taking into account existing wear models, Meng and Ludema [10] proposed to abandon efforts to model wear in terms of known wear mechanisms and develop full descriptions of the progression of macroscopic events on sliding surfaces. 


$\begin{array}{ll}\text { Nomenclature } \\ F_{\mathrm{a}} \quad \text { adhesive force } \\ F_{\mathrm{N}} \quad \text { normal contact force } \\ F_{\mathrm{nt}} \quad \text { normal joint force } \\ F_{\mathrm{s}} \quad \text { joint shear force } \\ F_{\mathrm{d}} \quad \text { dissipative contact force } \\ F_{\mathrm{r}} \quad \text { repulsion force } \\ K & \text { contact stiffness } \\ M & \text { mean particle mass }\end{array}$

This included a description of the formation and movement of fragmented particles in the interface region. This recalls the work of Godet and co-workers [11-13], who developed the concept of the third body in the 1970s, aware of the lack of conceptualization concerning dry contacts. The underlying idea was to use the same approach in order to study lubricated and dry contact. For this, Godet defines the third body as the medium at the interface of two bodies in contact. To study the behavior of the third body inside and outside the contact, Berthier proposed [14] the tribological circuit which brings into play all the third body flows and thus provides a very general view of the problem. This makes it applicable to a wide range of tribological situations. Based on this tribological circuit and coupling DESs to experimental, but simplified, wear studies, Fillot et al. [15-17] proposed a set of equations that allows a qualitative modeling of wear as a mass balance in the contact area. Iordanoff and Charles [18] showed how abrasion process can be studied as a particular and controlled wear process. Two-dimensional DESs were used. This study outlined the effects of abrasion process (free or fixed abrasive, re-circulating or loosed abrasive flow) on both removal material rate and sub-surfaced damage. This preliminary work has been carried out to outline the possibility of using DEM simulation for the study of SSDs. The model is based on micro-mechanical laws. The laws chosen for this study have been greatly simplified. The next step is to find the contact laws and link laws that allow a quantitative comparison between the presented results and classical results from the fracture mechanics literature [19], like the linear elastic F.M. Such comparison between discrete approach and continuous approach has been carried out for the study of silicon carbide by Ippolito et al. [20].

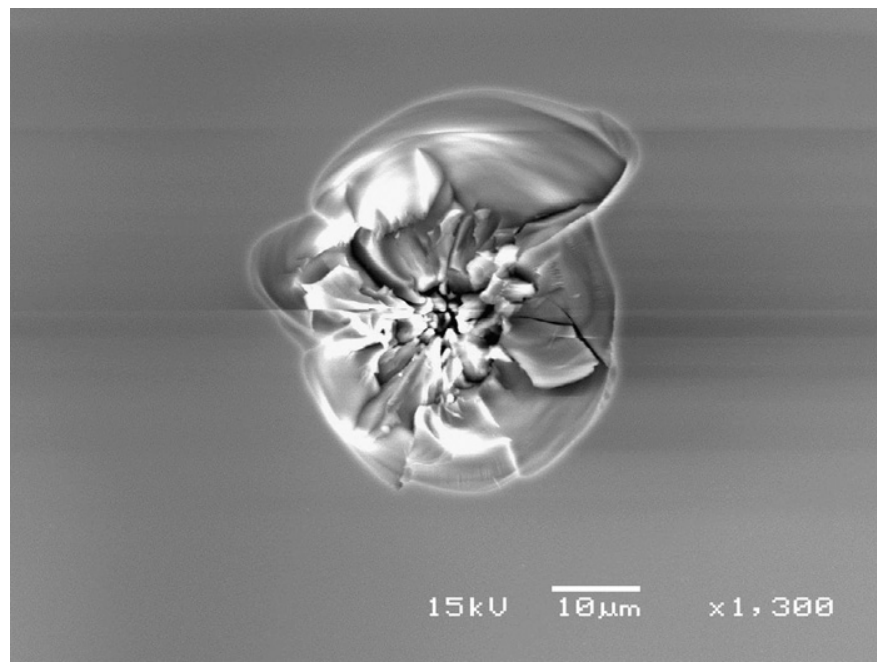

Fig. 1. Laser damage on a fused silica polished window created at $15 \mathrm{~J} / \mathrm{cm}^{2}, 3 \mathrm{~ns}$ pulse length.

$\begin{array}{ll}M_{i} & \text { mass of the sphere } i \\ M_{i j} & \text { equivalent mass } \\ N_{\mathrm{bj}} & \text { number of broken joint } \\ N_{\mathrm{d}} & \text { number of detached particles } \\ P_{\mathrm{N}} & \text { normal applied pressure } \\ R & \text { mean particle radius } \\ R_{i} & \text { particle radius of sphere } i \\ V_{\mathrm{p}} & \text { lower wall velocity, sliding speed } \\ \alpha & \text { viscous damping coefficient } \\ \delta & \text { overlap between two spheres }\end{array}$

\section{Numerical model}

The model of a degradable material has been widely described in Refs. $[15,16]$. The main principles are briefly recalled.

\subsection{Geometrical description}

According to Fig. 2, the upper first body (silica piece to be surfaced) is constituted by spheres linked together by elastic solid joints. The diameter of a given sphere is chosen randomly from a normal distribution around a mean diameter to avoid defining a specific layout for the spheres. This first body is linked to the upper wall.

The lower first body (tool) is simply defined as a lower rigid wall made of adjacent spheres. The roughness of the tool surfaced is linked to the sphere diameters.

The abrasive particles are spheres or group of spheres (one to four spheres) linked by an elastic solid joint.

\subsection{Boundary conditions}

Each wall has a single degree of freedom that allows translation. The upper plate is subjected to a constant normal pressure $P_{\mathrm{N}}$ along $z$ and is free to move vertically while the lower one moves horizontally with an imposed velocity $V_{\mathrm{p}}$ along $x$. Periodical boundary conditions are applied on the sides in order to mimic an infinite flow field along $x$.

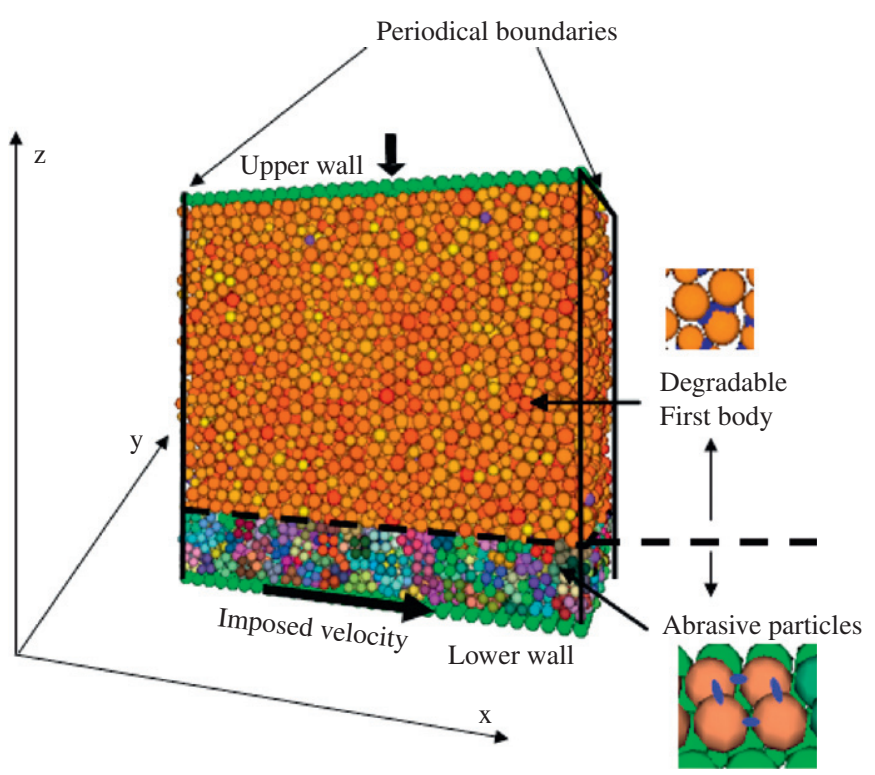

Fig. 2. Description of the simulated domain. 


\subsection{Particle interaction forces}

The particle interaction laws define the micro-mechanical properties of the media. These laws can be linked to the macroscopic rheological behavior of the material. The interaction forces are divided into contact and link forces. Every particle is submitted to contact force. This force acts only when two particle are in a compressive state. Two adjacent particles belonging to the same solid (silica first body or abrasive grain) are linked by a solid joint that acts in traction and shear state.

\subsubsection{Contact force}

Three types of forces are taken into account during the contact between two spheres: repulsion, adhesion (both are energy conservative) and energy dissipation. Simple interaction laws have been chosen. These three interactions are not linked in order to be able to identify the effect of each component on the global behavior. The knowledge of geometrical compressive interaction $\delta_{\text {nc }}$ allows the force calculation (Fig. 3).

- Repulsion is represented by a linear spring, whose stiffness is defined by $K$ and the force $F_{\mathrm{r}}$ corresponding to an overlap $\delta_{\mathrm{nc}}$ is

$F_{\mathrm{r}}=K \times \delta_{\mathrm{nc}}$

- Adhesion has been simplified to a constant $\gamma$ :

$$
F_{\mathrm{a}}=\gamma
$$

- The energy dissipation in the contact is due to viscous damping, and the damping force is written classically as:

$$
F_{\mathrm{d}}=2 \alpha \sqrt{K M_{i j}} \times \dot{\delta}_{\mathrm{nc}}
$$

where $\alpha$ is the damping coefficient $(<1), \dot{\delta}_{\mathrm{nc}}$ the impact speed, and $M_{i j}$ is the equivalent mass of the contact:

$\frac{1}{M_{i j}}=\frac{1}{m_{i}}+\frac{1}{m_{j}}$

The sum of the interaction forces is

$$
\begin{aligned}
& \vec{F}_{\text {contact }}=\vec{F}_{\mathrm{r}}+\vec{F}_{\mathrm{a}}+\vec{F}_{\mathrm{d}} \\
& F_{\text {contact }}=-K \delta_{\mathrm{nc}}+\gamma-2 \alpha \sqrt{K M_{i j}} \times \dot{\delta}_{\mathrm{nc}}
\end{aligned}
$$

where $F$ and $\dot{\delta}_{\text {nc }}$ are algebraic values.

This type of law is commonly used in DEM simulations. One of the main advantages is that it provides a direct link between the viscous damping coefficient and the coefficient of restitution used in kinetics models $[21,22]$.

\subsubsection{Solid joint law}

The solid link is created between two adjacent spheres belonging to the same solid. The normal force due to this link is

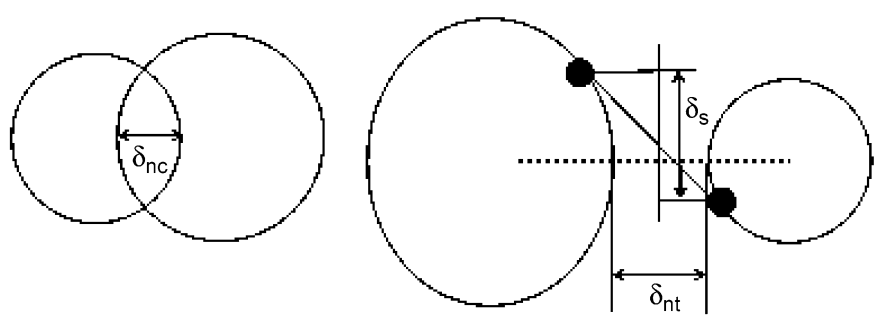

Fig. 3. Contact and joint definition. calculated only under traction state (Fig. 3) and is equal to

$F_{\mathrm{nt}}=K \times \delta_{\mathrm{nt}}$

The compressive force is calculated when contact occurs. This way to calculate normal force allows taking into account a difference between compressive and traction stiffness for a given material. In the present study, compressive and traction stiffness are the same.

The shear force (Fig. 3) is calculated, thanks to the shear distance calculated between two points at the contact sphere periphery:

$F_{\mathrm{s}}=K \times \delta_{\mathrm{s}}$

\subsubsection{Particle detachment}

In this simulation, the links between abrasive particles are non-breakable.

For silica, the yield stress under traction ( $50 \mathrm{MPa}$ ) is far under the yield stress under compression (1050 MPa). In the present study, it is assumed that first body (silica) link breaks only under traction state: when the link is under traction and when the link force reaches a yield value $F_{\mathrm{y}}$, the link is broken. This law is characteristic of a brittle material.

\subsection{Numerical algorithm}

An explicit algorithm is chosen. The progression of the sample requires cyclic calculations. Given the particle's centers positions at time $t, \mathbf{P}_{i}^{t}\left(x_{i}^{t}, y_{i}^{t}\right)$, all inter particle contacts can be identified and their corresponding overlap $\delta$ calculated. It is then possible to compute the inter-particle forces using a given force-displacement law. The knowledge of particle rotation at time $t$ allows calculating the joint forces. Once the forces are calculated, Newton's second law enables the computation of the translation accelerations $\mathbf{A}_{i}^{t}\left(\ddot{x}_{i}^{t}, \ddot{y}_{i}^{t}, \ddot{z}_{i}^{t}\right)$ of the particle centers and particle rotation acceleration $\theta_{i}^{t}\left(\ddot{\theta}_{x i}^{t}, \ddot{\theta}_{y i}^{t}, \ddot{\theta}_{z i}^{t}\right)$ :

$\ddot{x}_{i}^{t}=\sum \frac{F x_{j \rightarrow i}}{m_{i}}, \quad \ddot{y}_{i}^{t}=\sum \frac{F y_{j \rightarrow i}}{m_{i}}, \quad \ddot{z}_{i}^{t}=\sum \frac{F z_{j \rightarrow i}}{m_{i}}$

where $m_{i}$ is the particle mass.

$\ddot{\theta}_{x i}^{t}=\sum \frac{M_{x_{j \rightarrow i}}}{I_{x i}}, \quad \ddot{\theta}_{y i}^{t}=\sum \frac{M_{y_{j \rightarrow i}}}{I_{y i}}, \quad \ddot{\theta}_{z i}^{t}=\sum \frac{M_{z_{j \rightarrow i}}}{I_{z i}}$

where $I_{x i}, I_{y i}$, and $I_{z i}$ are the particle inertia around $G_{x}, G_{y}$ and $G_{z} . G$ is the particle center of gravity.

Finally, a Verlet velocity algorithm [23], which is a classical explicit algorithm in DEM, is used to integrate the velocities of the sphere centers, the rotational velocities, the new positions of sphere centers and the new position of the solid joint action points over a small time step $\mathrm{d} t$.

This method of computation is rather simple. On the other hand, the time step must be chosen with care: it has to be short enough to localize all the inter-particle contacts and long enough to avoid prohibitive computer times.

\subsection{Numerical sample}

The sample is generated by a random creation of spheres (Fig. 4a). Then, the domain is compressed and links are created each time a contact is detected (Fig. 4b). No link is created with the lower wall. The body created by this assembly of random sphere will be the same for all simulations. The virtual building of the first body is commonly referred to as 'numerical sintering'. 
a

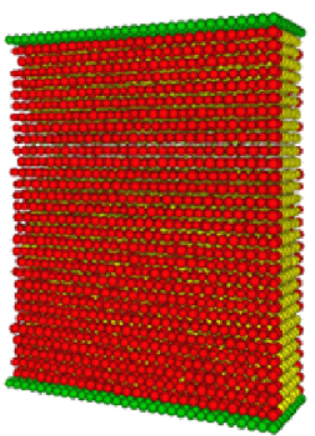

$\mathrm{b}$

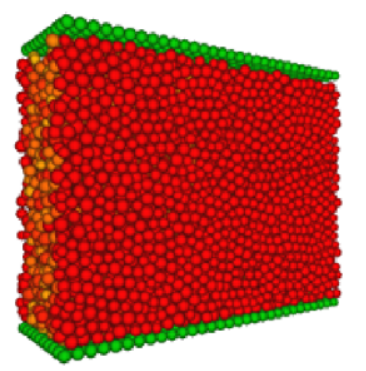

C

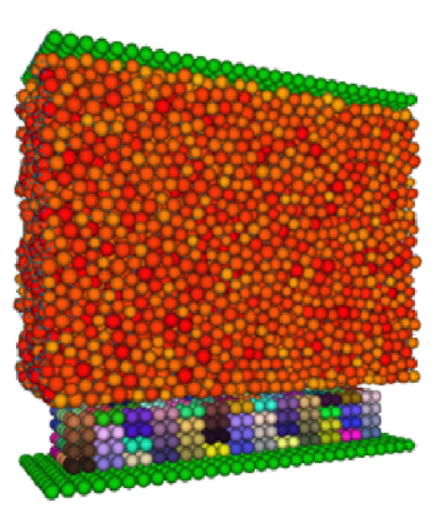

d

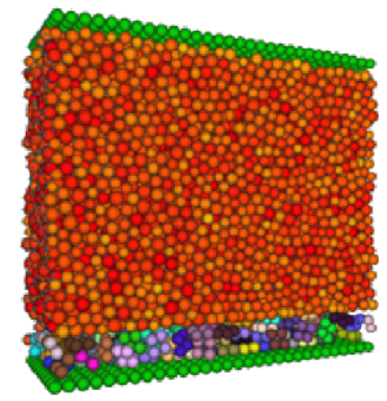

Fig. 4. Steps to obtain the numerical sample.

Then abrasive particles are created between the lower wall and the first body (Fig. 4c). The domain is compacted once again till the nominal compressive pressure is reached (Fig. 4d). This last state defines the initial domain for abrasion study.

\subsection{Choice of dimensionless parameters}

In a first qualitative study, dimensionless parameters have been chosen. They allow a direct comparison between the input parameters.

By taking $\sqrt{M / K}$ as the time scale, the mean particle mass $M$ as the mass scale and the mean particle radius $R$ as the length scale, the dimensionless pressure, velocity and adhesion are then directly linked to the characteristic overlaps:

$\bar{P}_{\mathrm{N}}=\frac{P_{\mathrm{N}} R}{K}=\frac{\delta_{\mathrm{c}}}{R}=\bar{\delta}_{\mathrm{c}}$

$\bar{V}_{\mathrm{p}}=\frac{V_{\mathrm{p}}}{R} \sqrt{\frac{M}{K}}=\frac{\delta_{\mathrm{o}}}{R}=\bar{\delta}_{\mathrm{o}}$

$\bar{\gamma}=\frac{\gamma}{K R}=\frac{\delta_{\mathrm{eq}}}{R}=\bar{\delta}_{\mathrm{eq}}$

The other dimensionless parameters are

$\bar{R}_{i}=\frac{R_{i}}{R}, \quad \bar{M}_{i}=\left(\frac{R_{i}}{R}\right)^{3}, \quad \bar{K}=1$

where $R_{i}$ and $M_{i}$ are the radius and the mass of the sphere $i$, respectively.

The dimensionless force is given by

$\bar{F}=\frac{F}{K R}$

Some parameters such as the damping coefficient are already dimensionless.

\subsection{Scope and limits of the study}

\subsubsection{Time}

The oscillating period of a non-damped contact is proportional to

$T=\sqrt{\frac{M}{K}}$

where $M$ is the mean mass of the spheres.

The time step $\mathrm{d} t$ of the numerical simulation must be a fraction of $T$ :

$\mathrm{d} t=\frac{1}{N} \sqrt{\frac{M}{K}}$

with $N$ being an integer chosen equal to 10 in the following simulations.

Dimensionless time step is

$\mathrm{d} \bar{t}=\frac{1}{N}$

\subsubsection{Overlaps}

In order to neglect the granular contact vibration problem with respect to the macroscopic tribological problem, the contact oscillation period has to be very small with respect to the time spent for a sphere to move over distance $R$ at speed $V_{\mathrm{p}}$ :

$\sqrt{\frac{M}{K}} \ll \frac{R}{V_{\mathrm{p}}}$

Condition (15) associated with the expression for dimensionless velocity $\bar{V}_{\mathrm{p}}(9)$ leads to

$\bar{V}_{\mathrm{p}}=\bar{\delta}_{\mathrm{o}} \ll 1$

To remain in the realm of non-deformable solid mechanics, the static overlaps due to pressure and adhesion must remain negligible compared to the radius of a sphere. This condition is written as

$\bar{\gamma}=\bar{\delta}_{\text {eq }} \ll 1$ and $\quad \bar{P}_{\mathrm{N}}=\bar{\delta}_{\mathrm{c}} \ll 1$

\section{Numerical simulation}

\subsection{Numerical data}

The goal of this study is to give some first qualitative results concerning abrasion process during grinding of silica windows. A first work is to determine the micro-mechanical properties of the numerical sample, thanks to macroscopic mechanical properties.

\subsubsection{Contact and joint stiffness evaluation}

Once the young modulus $E$ is known, the particle stiffness is chosen in order to fit the normal stiffness of an elementary cylinder which diameter is equal to the particle diameter. For this, let us consider a column of spheres under compression (Fig. 5).

Each sphere is submitted to a normal stress $\sigma$ defined by

$\sigma=\frac{F}{S}=E \times \frac{\Delta L}{L}=E \varepsilon$

where $\Delta L / L=\varepsilon$ can be define as the normal strain for one sphere, $E$ is the young modulus, and $S$ is the sphere area.

Let us consider that the $(n+1)$ spheres are linked by $n$ springs. If $k$ is the spring stiffness and $F$ is the compressive force, the relationship between the compressive force and the total displacement is

$F=K_{\mathrm{eq}} \times \Delta L=\frac{K}{n} \Delta L$ 


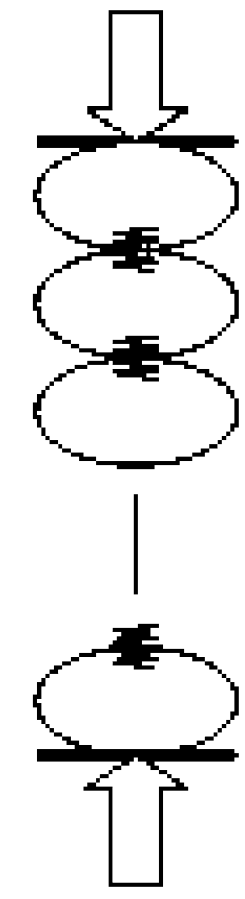

Fig. 5. Model for the compression of one column of spheres

Dividing by the sphere area $S$,

$\frac{F}{S}=\frac{L K}{n S} \times \frac{\Delta L}{L}$

This can be written as

$\sigma=E \varepsilon$

With $L=2 n R$ and $S=(2 R)^{2}$,

$K=2 R E$

\subsubsection{Dimensionless parameters}

With the help of Eqs. (9) and (22), the dimensionless normal pressure and shear velocities are given by

$\bar{P}=\frac{P R}{2 R E}=\frac{P}{2 E}$

$\bar{V}=V \times \sqrt{\frac{2 \pi}{3}} \times \sqrt{\frac{\rho}{E}}$

with $M=\rho \times(4 / 3) \pi R^{3}, \rho$ is the density of the media.

The yield force is found using Eqs. (11) and (22):

$\bar{F}_{\mathrm{y}}=\frac{F_{\mathrm{y}}}{2 R E \times R}$

and $F_{\mathrm{y}} / S=\sigma_{\mathrm{y}}$, where $\sigma_{\mathrm{y}}$ is the material yield stress and $S=(2 R)^{2}$ is the projected area for one sphere. Finally,

$\bar{F}_{\mathrm{y}}=\frac{2 \sigma_{\mathrm{y}}}{E}$

\subsubsection{Dimensionless data}

The material considered in this study is SUPRASIL 312. The mechanical properties of this material are [24]:

$\begin{array}{ll}\text { Density }\left(\mathrm{kg} / \mathrm{m}^{3}\right) & 2201 \\ \text { Young modulus }\left(\mathrm{N} / \mathrm{m}^{2}\right) & 7.25 \mathrm{E} 10 \\ \text { Yield tensile stress }\left(\mathrm{N} / \mathrm{m}^{2}\right) & 50 \mathrm{E} 6\end{array}$

The density of the granular media equals the sphere density times the volume fraction. The volume fraction of our numerical sample is 0.65 . Then, the sphere density will be taken equal to the SUPRASIL density divided by 0.65 .

The order of magnitude for the shear velocity for surfacing process is $1 \mathrm{~m} / \mathrm{s}$. The order of magnitude for the applied pressure is $1 \mathrm{kPa}$ [8].

This gives the following dimensionless data for the simulations:

$\bar{P}=7 \times 10^{-9}$

$\bar{V}=3 \times 10^{-4}$

$\bar{F}_{\text {rupt }}=1.38 \times 10^{-3}$

The simulation requires a high number of iterations: typically $1 / \bar{V}$ iterations for a tool displacement equals to a sphere radius. This number is then inversely proportional to the velocity. Then, it is chosen to increase the shear velocity in order to keep the condition defined in Eq. (16):

$\bar{V}=1 \times 10^{-2}$

This value corresponds to a shear velocity equals to $30 \mathrm{~m} / \mathrm{s}$.

Detachment rate is also very sensitive to pressure [16]. To increase this rate and obtain reasonable calculation time, a pressure equals to $1 \mathrm{MPa}$ is chosen, that gives a dimensionless pressure equals to $7 \times 10^{-6}$

\subsubsection{Abrasive domain}

The main effect studied in this preliminary work is abrasive size and density. The abrasive is obtained, thanks to the association of spheres. Generally, abrasive particles present profiles with angularity that cause high local stress and creates damages. The choice of spheres with little radius is able to create such effect. In this study, particles from the abrasive and particles from fused silica have comparable sizes and this effect is not studied.

Table 1 summarizes the different abrasive size and quantity used in the simulations. Abrasive shape is a plate formed by an assembly of four spheres. To study the effect of abrasive particle size, two sizes are chosen: for 'little' abrasive, the sphere diameter is 2 (same than the mean sphere diameter for the silica); for 'big' abrasive, the sphere diameter is 4 . To study the effect of the number of abrasive particle through the contact, two cases are chosen. For the case called 'Discrete', the number of abrasive particle is chosen in order to separate the silica from the tool by few abrasive particles. The 'LayerOf' case corresponds to a number of particles that allow one "continuous layer of abrasive particle in the contact. Fig. 6 shows a draw for the initial state of the four tested configurations.

\subsubsection{Re-circulating flow}

Once a particle of silica is detached, particles can exit the contact through the side normal to the shear direction. This case simulates the contact feeding with new abrasive that contains no detached particles from silica.

\subsection{Numerical results}

First the number of detached particles and the number of broken joint versus time are plotted. Fig. 7 shows two domains. In the beginning of simulation, all the curves have the same behavior. In that part, one can think that in all cases, existing defaults inside the material conduce to the same particle detachments and broken joints whatever abrasive property is chosen. The detachment rate and broken joint rate in that part is 
much higher than in the following. In a second part, the detachment velocity is greatly influence by the size and quantity of abrasive. The repartition of abrasive on the whole first body surface decreases the effects of force localization. The higher detachment velocities are obtained for bigger abrasive particles and less abrasive density. The case 'DiscreteBig' that correspond to six plates of big particles gives the higher detachment rate. The number of broken joint seems to be correlated to the number of detached particles as is confirmed by Fig. 8 .

Fig. 8 gives first information on broken joint number necessary to particle detachment. To obtain a minimum amount of residual cracks inside the silica, one can think that detachment may occur with a minimum number of broken joints. The ideal case should be to break only the joints that lead to detachment. As the mean number of joint per particle is 5 , the ideal ratio between broken joint $N_{\mathrm{bj}}$ and particle detachment $N_{\mathrm{d}}$ should be 5 . This curve does

Table 1

Summary of the abrasive used in simulation

\begin{tabular}{lcll}
\hline & $\begin{array}{l}\text { No. of } \\
\text { abrasive } \\
\text { spheres }\end{array}$ & $\begin{array}{l}\text { Size of } \\
\text { spheres }\end{array}$ & Abrasive morphology \\
\hline Case 'DiscreteBig' & 24 & 4 & Plate of four spheres \\
Case 'LayerOfLittle' & 156 & 2 & Plate of four spheres \\
Case 'DiscreteLittle' & 76 & 2 & Plate of four spheres \\
Case 'L.ayerOfbig' & 96 & 4 & Plate of four spheres \\
\hline
\end{tabular}
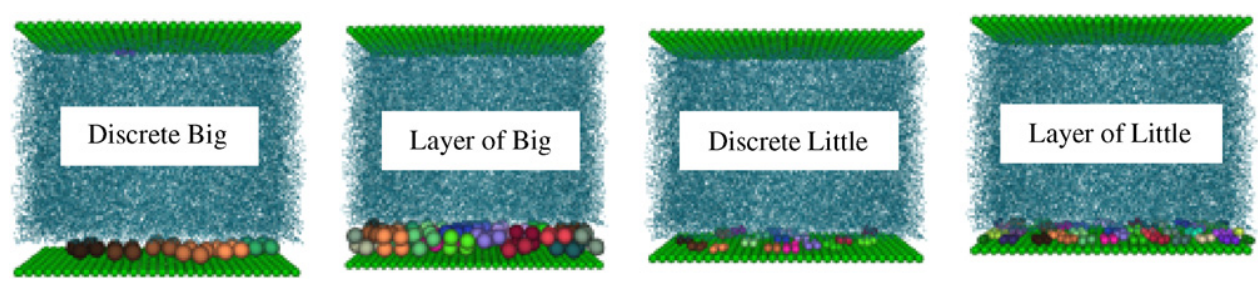

Fig. 6. Description of the abrasive used in simulations.
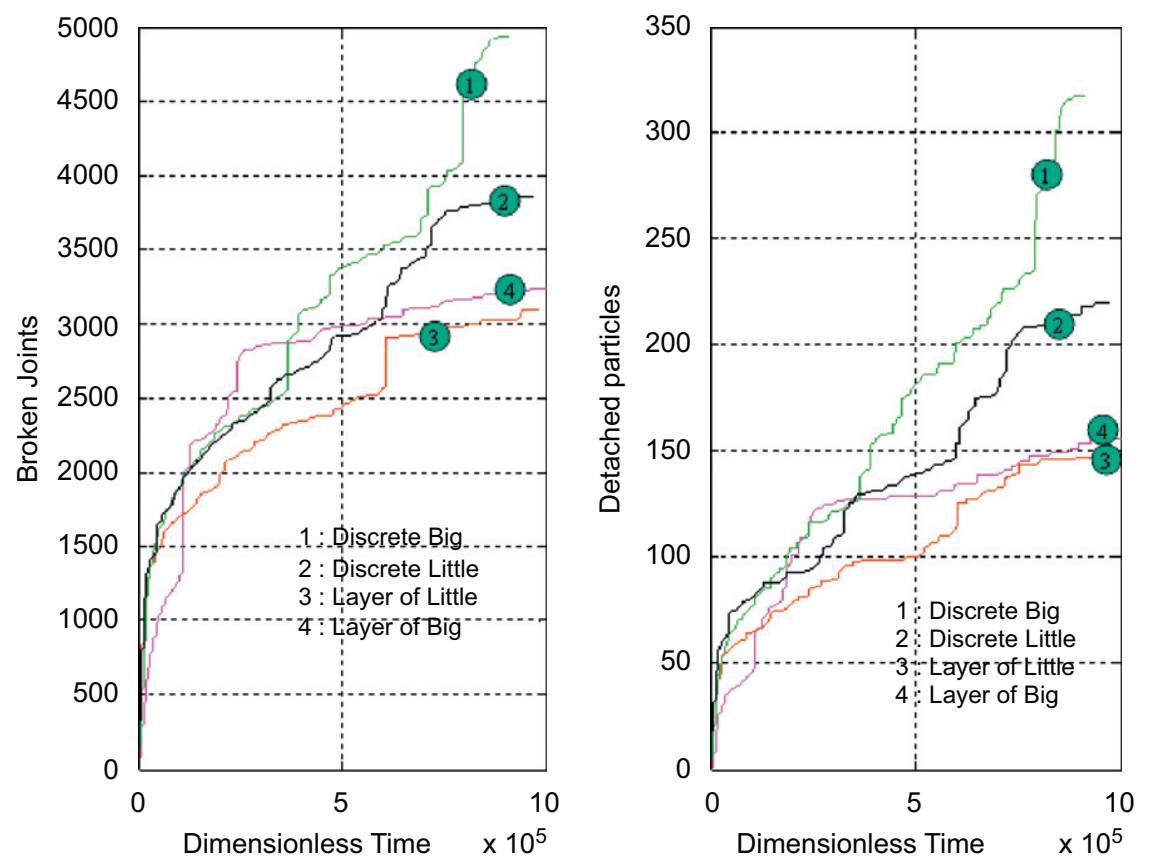

Fig. 7. First body degradation versus time. 
abrasive size on residual cracks has been experimentally studied. The number of dark spot analyzed by this group at different depths can be related to the default (broken joint) in the present simulation. A second important result is the effect of abrasive size that seems to be the dominant parameter concerning SSDs. The number of damages, between the thicknesses 5 and 15 is twice to 10 times higher with bigger abrasive particles. Once again, qualitatively, this important result is in accordance with the experimental result published in Ref. [9], where $15 \mu \mathrm{m}$ loose abrasive creates much more default than the $7 \mu \mathrm{m}$ one.

Finally, detachment rate and SSDs are not completely correlated. As a matter of fact, the density of abrasive seems to be the dominant parameter for detachment rate while abrasive size is the dominant parameter for sub-surface damage.

\section{Conclusion}

SSD is known from the literature to be the main cause of damages in fused silica optics used in laser mega joule facilities. One of the possible causes of SSDs is supposed to be surfacing

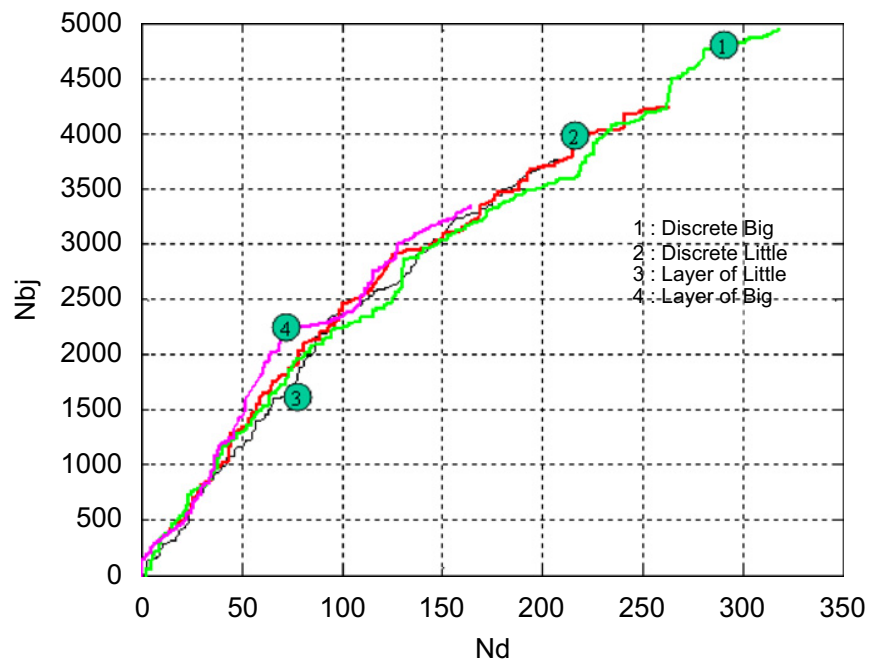

Fig. 8. Particle detachment versus broken joint. processes. These sub-surface damages are difficult to experimentally described and predict. It is proposed to develop a numerical tool as a help to investigate relationship between SSDs and abrasion process. A DES has been developed to simulate the silica, the abrasive particles, the detached particles and the cracks inside the surfaced silica. Adapted from wear study, this simulation is developed to investigate SSDs. A first model for silica and abrasive is proposed. Four different cases of abrasion are presented. They focus only on abrasive influence: the choice of abrasive to decrease SSDs is one of the known key parameters. First results shows how this numerical tool allows obtaining a local analyze of SSD. These results qualitatively confirm experimental works published in this domain, in terms of SSD description and abrasive size effect.

This first work shows the interest of the discrete element tool when creation of a great number of sub-surface micro-crack formations has to be simulate. This tool must be improved in mainly three directions:

- Model for fused silica: The chosen perfectly brittle joint law should be improved in order to link micro-mechanical law to macroscopical fracture theory for brittle materials.

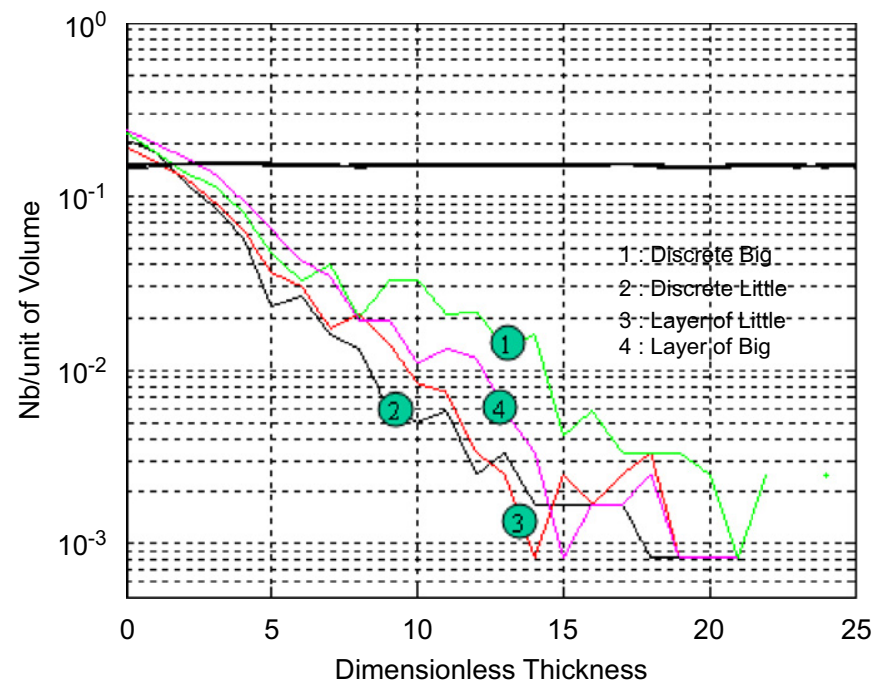

Fig. 10. Comparison of SSD at time corresponding to 150 detached particles.

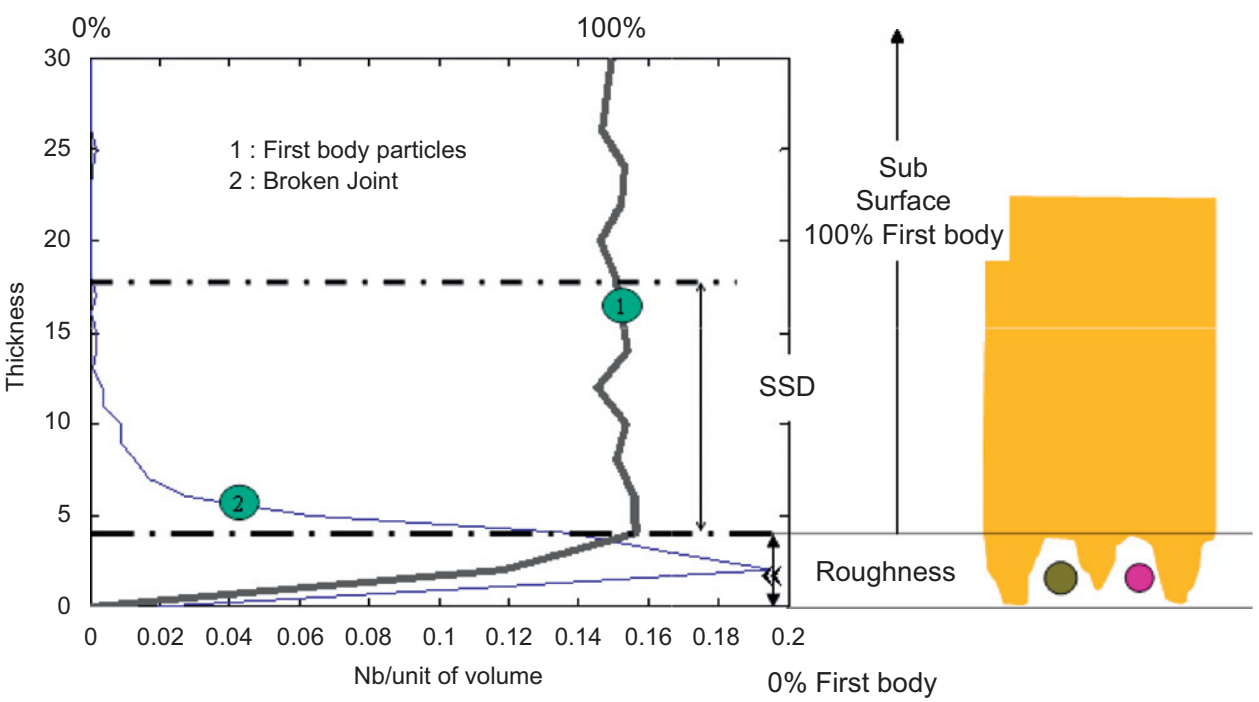

Fig. 9. Sub surface damage for case 'LayerOfLittle' at time $3.10^{4}$. 
- Model for abrasive particles: Different shapes should be developed. Fixed and loose abrasive should be taken into account and compared.

- Improve calculation speed in order to be able to simulate abrasion process with mechanical parameters close to the nominal parameters found in the literature.

\section{References}

[1] André ML. Status of the LMJ project. In: André ML, editor. Solid state lasers for application to inertial confinement fusion, Second annual international conference. Proc SPIE 1996;3047:38-42.

[2] Lowdermilk WH. Status of the National Ignition Facility project. In: André ML, editor. Solid state lasers for application to inertial confinement fusion, Second annual international conference. Proc SPIE 1996;3047:16-37.

[3] Bercegol H, Bouchut P, Lamaignere L, Le Garrec B, Raze G. The impact of laser damage on the lifetime of optical components in fusion lasers. In: Exarhos GJ, Guenther AH, Kaiser N, Lewis KL, Soileau MJ, Stolz CJ, editors. Proceedings of laser-induced damage threshold in optical materials 2003. Proc SPIE 2004; 5273:312-24.

[4] Menapace JA, Penetrante B, Golini D, Slomba AF, Miller PE, Parham TG, Nichols M, Peterson J. Combined advanced finishing and UV-laser conditioning for producing UV damage resistant fused silica optics. In: Exarhos GJ, Guenther AH, Kaiser N, Lewis KL, Soileau MJ, Stolz CJ., editors. Proceedings of laser-induced damage threshold in optical materials. Proc SPIE 2001; 4679:56-68.

[5] Camp DW, et al. Subsurface damage and polishing compound affect the $355 \mathrm{~nm}$ laser damage threshold of fused silica surfaces. In: Proceedings of laser-induced damage threshold in optical materials. Proc SPIE 1998;3244:356-64.

[6] Genin FY, Salleo A, Pistor TV, Chase LL. Role of light intensification by cracks in optical breakdown on surfaces. J Opt Soc Am A 2001;18(10).

[7] Feit MD, Rubenchik AM. Influence of subsurface cracks on laser induced surface damage. In: Exarhos GJ, Guenther AH, Kaiser N, Lewis KL, Soileau MJ, Stolz CJ, editors. Proceedings of laser-induced damage threshold in optical materials 2003. Proc SPIE 2004;5273:264-72.
[8] Karow HK. Fabrication methods for precision optics. Wiley Interscience; 1993.

[9] Sutatwala T, Wong L, Miler P, Feity MD, Menapace J, Steele R, et al. Sub-surface mechanical damage distribution during grinding of fused silica. J Non-Cryst Sol 2006;352:5601-17.

[10] QUOTE Meng HC, Ludema KC. Wear models and predictive equations: their form and content. Wear 1995;181-183:443-57.

[11] Godet M. The third body approach: a mechanical view of wear. Wear 1984;100:437-52.

[12] Godet M. Third-bodies in tribology. Wear 1990;136:29-45.

[13] Ludema KC. Third Bodies: Perspectives on modeling in lubricated contacts. In Close fitting contacts, etc: following on the concepts of Dr. Maurice Godet Proceedings of the 22nd Leeds-Lyon symposium on tribology. Elsevier Tribol Ser 1995;31:3-19.

[14] Berthier Y. Maurice Godet's third body. In: Proceedings of the 22nd LeedsLyon symposium on tribology. Tribol Ser 1995;31:21-30.

[15] Fillot N, Iordanoff I, Berthier Y. A granular dynamic model for the degradation of material. ASME J Tribol 2004;126:606-14.

[16] Fillot N, Iordanoff I, Berthier Y. Kinetics of particle detachment: contribution of a granular model. Tribol Ser 2004;43:63-73.

[17] Fillot N, Iordanoff I, Berthier Y. Simulation of wear through mass balance in a dry contact. ASME J Tribol 2005;127:230-7.

[18] Iordanoff I, Charles JL. Discrete element method: an helpful tool for abrasion process study. In: Proceedings of the I MECH E Part B. J Eng Manufact 2007;221(6):1007-19.

19] Ceriolo L, Di Tommaso A. Fracture mechanics of brittle materials: a historica point of view. In: Proceedings of the 2nd international Ph.D. symposium in civil engineering, Budapest, 1998.

[20] Ippolito M, Mattoni A, Colombo L. Role of lattice discreteness on brittle fracture: atomistic simulations versus analytical models. Phys Rev B 2006; 73:104111.

[21] Iordanoff, I, Khonsari MM. Granular lubrication: towards an understanding of the transition between kinetic and fluid regime. ASME J Tribol 2004;126: 137-45.

[22] Mishra BK, Murty CVR. On the determination of contact parameters for realistic DEM simulations of ball mills. Powder Technol 2001;115:290-7.

[23] Verlet L. Computer "Experiments" on classical fluids. I. Thermodynamical properties of Lennard-Jones molecules. Phys Rev 1967;159:98-103.

[24] Quartz glass for optics. Brochure, Heraeus, Data and properties. 